\title{
Mass spectrometry mapmaking
}

A comprehensive mass spectrometric map of the yeast proteome is a valuable resource for both discovery-based and targeted proteome analyses.

In choosing targeted proteome analysis by mass spectrometry as our 2012 Method of the Year, we noted the need for comprehensive mass spectrometry 'assays' for detecting and quantifying specific proteins. Such a resource could enable the broader community to utilize the technology to follow sets of proteins of interest with high sensitivity.

Ruedi Aebersold and Paola Picotti of ETH Zurich, along with Andreas Beyer of Technische Universität Dresden, and their colleagues now present a nearly complete mass spectrometric 'map' of the yeast proteome. The map, the first of its kind for any proteome, comes in two versions, supporting both targeted proteome analysis and discovery-based proteomics.
To generate this map, the researchers began with the 6,607 protein sequences predicted from the Saccharomyces cerevisiae genome. For each protein that could be found in the proteomic database PeptideAtlas, they identified a set of up to eight 'proteotypic' peptides with unique sequences and favorable mass spectrometry properties. For those proteins that had not been previously detected, they predicted proteotypic peptides. They made synthetic versions of the compiled proteotypic peptides, 28,000 in all, accounting for $97 \%$ of the yeast proteome.

Peptides in hand, the researchers analyzed them using a linear ion trap-Orbitrap hybrid instrument to generate fragment-ion spectra. This reference spectral library can be used to interpret experimental spectra in discovery-based experiments. They also analyzed the peptides on a triple quadrupole mass spectrometer to establish coordinates for selected reaction monitoring. These coordinates were used to develop mass spectrometry assays to detect specific proteins of interest in targeted proteomics analyses.

The team showed the worth of this map by performing a quantitative trait locus analysis, which correlates protein abundance with genetic variation. This type of analysis requires that protein concentrations be precisely measured in a large number of samples. With the help of the map, they detected instances of co-inheritance of genetic variations in yeast that influence related protein abundances.

The map is available in PeptideAtlas and can be found at http://www.srmatlas.org/ yeast/.

\section{Allison Doerr}

\section{RESEARCH PAPERS}

Picotti, P. et al. A complete mass-spectrometric map of the yeast proteome applied to quantitative trait analysis. Nature advance online publication (20 January 2013). 